\title{
Pion Form Factor Contribution in Vacuum Polarization corrections for 1s energy level in different Pionic-Hydrogen Atoms
}

\author{
M El Shabshiry ${ }^{1}$, S. Ismaeel ${ }^{2}$ \\ ${ }^{1}$ Department of Physics, Faculty of Science, Ain Shams University 11556, Cairo, Egypt \\ ${ }^{2}$ College of Sciences and Humanities, Prince Sattam Bin Abdulaziz University, Kharj, Saudi Arabia
}

\begin{abstract}
The 1 s energy level vacuum polarization correctionsof pionic hydrogen atom induced by a potential including form factor are compared with those obtained by using pion point potential. Without form factor of nucleus and pion the correction increases very slowly for low $Z$ atoms and increases fastly for higher $Z$. The finite size of the nucleus increases the correction with $Z$ in case of exponential distribution, while in case of Gaussian distribution the increase is lower. For Fermi distribution there is a fast increase at low values of $Z$ and faslty decreases with higher values of $Z$. The effect of form factor of pion on the correction is very clear for low $Z$ nuclei and then becomes nearly constant for higher values $Z$.
\end{abstract}

Keywords: Vacuum polarization correction, pion form factor, pionic-hydrogen atom.

\section{Introduction}

Vacuum polarization plays an important role of calculating the energy levels of atoms. It is small in hydrogen atoms but for heavier orbital particles such as muons and pions it gives a dominant contribution to QED corrections. It is the interaction of the bound electron with the electrons in the Dirac Sea.

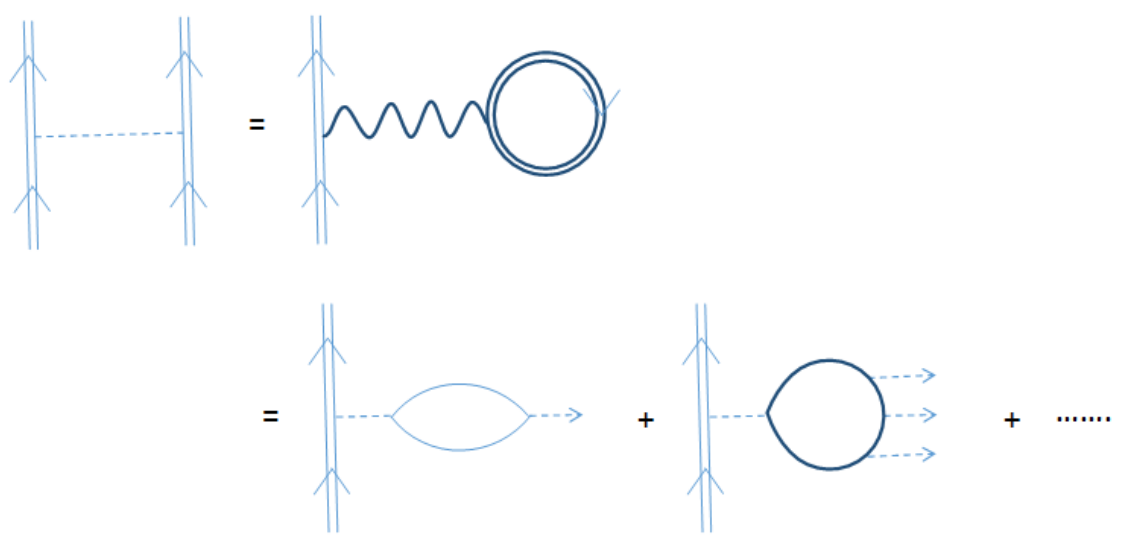

Fig.1 Represents Feynman diagram for one loop vacuum polarization.

We calculated the vacuum polarization corrections in different energy levels for mionic-hydrogen atom [1] and for pionic-hydrogen atom with finite size proton [2].The results are very near from those calculated by Borie [3].In this work we study the effect of pion form factor in 1s level for different pionic-hydrogen atoms. In these calculations the relativistic approach is used. Borie [3] calculated the Lamb shift in case of mionichydrogen atom. The mionic hydrogen $(2 p-2 s)$ transition was investigated by Carroll et.al. [4]. They used a precise non-perturbative numerical solution of the Dirac equation including the finite size Coulomb force and finite size vacuum polarization.Lee et.al. [5] derived a formula for the vacuum polarization correction for the spin zero orbital particle. They used the formula for these corrections based on Klein-Gordon-Fock equation.Karshenbiomet. al. [6] used the Uehling potential for calculating one loop vacuum polarization for the pionic circular states. In this work we usethis formula to calculate the vacuum polarization corrections in $1 \mathrm{~s}$ state for different pionic-hydrogen atoms from $Z=1$ to $Z=68$. The main aim of this work is to show the effect of the pion form factor on these corrections for different pionic-hydrogen atoms.

\section{Theory}

The relativistic equation of Klein-Gordon-Fockwhich describes the spin zero particle, has the form

$\left(\left[E_{i}-V(\vec{r})\right]^{2}-P^{2} c^{2}-m_{\pi}^{2} c^{4}\right) \Phi_{i}(\vec{r})=0$ 
$\Phi_{i}(r)$ are not orthogonal and their normalization is not trivial. The solution of this equation with non-Coulomb potential is possible [7-8]. The perturbative theory of Coulomb potential has not been developed till now. The vacuum polarization corrections in energy levels are calculated by using a recent form of Lee et.al. [5]

Where

$$
\Delta E_{i}=\frac{\left\langle\Phi_{i}^{(o)}\left|\left(E_{i}^{(o)}-V^{(o)}\right) \delta V\right| \Phi_{i}^{(o)}\right\rangle}{\left\langle\Phi_{i}^{(o)}\left|E_{i}^{(o)}-V^{(o)}\right| \Phi_{i}^{(o)}\right\rangle}
$$

$$
\Phi_{n l}^{(o)}=\frac{1}{r} R_{n l}(r) Y_{l m}(\theta, \phi)
$$

Where $R_{n l}(r)$ is taken from [2]. In case of electrostatic potential this equation is valid for any perturbation such as Uehling corrections. The Coulomb problem is considered as an unperturbed case and we take the unperturbed solution of the last equation from [2]. The vacuum polarization perturbative potential for finite nuclei including the form factor of pion is obtained by folding the Uehling potential with the extended source distribution and the pion distribution, which has the form,

Where

$$
\delta V(r)=4 \pi \int \frac{d^{3} q}{(2 \pi)^{3}} \frac{e^{i \vec{q} \cdot \vec{r}}}{q^{2}} \Pi^{R}\left(-q^{2}\right) \rho_{N}(q) \rho_{\pi}(q)
$$

$\rho_{N}(q)=\int d^{3} r_{N} e^{-i \vec{q} \cdot \vec{r}_{N}} \rho_{N}\left(r_{N}\right), \quad \rho_{N}\left(r_{N}\right)=-Z e \rho\left(r_{N}\right)$

$\rho_{\pi}(q)=\int d^{3} r_{\pi} e^{-i \vec{q} \cdot \vec{r}_{\pi}} \rho_{\pi}\left(r_{\pi}\right) \quad, \quad \rho_{\pi}\left(r_{\pi}\right)=-e \rho\left(r_{\pi}\right)$

$\rho\left(r_{N}\right)$ is the central nuclear density and is taken in Gaussian, exponential, and Fermi forms and the density of pion, $\rho\left(r_{\pi}\right)$, is in Gaussian.

$$
\begin{aligned}
& \delta V(r)=\int d^{3} r_{N} \rho_{N}\left(r_{N}\right) \int d^{3} r_{\pi} \rho_{\pi}\left(r_{\pi}\right) \int \frac{d^{3} q}{(2 \pi)^{3}} e^{-i \vec{q} \cdot\left(\vec{r}-\vec{r}_{N}-\vec{r}_{\pi}\right)} \frac{4 \pi}{q^{2}} \Pi^{R}\left(-q^{2}\right)\left(-\frac{\alpha q^{2}}{15 \pi m^{2}}\right) \\
= & -\frac{4 \pi \alpha}{15 m^{2} \pi} \int d^{3} r_{N} \rho_{N}\left(r_{N}\right) \int d^{3} r_{\pi} \rho_{\pi}\left(r_{\pi}\right) \int \frac{d^{3} q}{(2 \pi)^{3}} e^{-i \vec{q} \cdot\left(\vec{r}-\vec{r}_{N}-\vec{r}_{\pi}\right)} \\
= & -\frac{4 \pi \alpha}{15 m^{2} \pi} \int d^{3} r_{N} \rho_{N}\left(r_{N}\right) \int d^{3} r_{\pi} \rho_{\pi}\left(r_{\pi}\right) \delta^{3}\left(\vec{r}-\vec{r}_{N}-\vec{r}_{\pi}\right) \\
= & -\frac{4 \pi \alpha}{15 m^{2} \pi} \int_{\infty}^{\infty} d^{3} r_{N} \rho_{N}\left(r_{N}\right) \rho_{\pi}\left(r-r_{N}\right) \\
= & -\frac{(4 \pi)^{2} \alpha}{15 m^{2} \pi} \int_{0}^{\infty} r_{N}^{2} d r_{N} \rho_{N}\left(r_{N}\right) \rho_{\pi}\left(r-r_{N}\right)
\end{aligned}
$$

Substituting the values of $\rho_{N}\left(r_{N}\right)$ and $\rho_{\pi}\left(r_{\pi}\right)$ we get

$$
\delta V(r)=-\frac{(4 \pi)^{2} Z \alpha^{2}}{15 m^{2} \pi} \int_{0}^{\infty} r_{N}^{2} d r_{N} \rho\left(r_{N}\right) \rho\left(r-r_{N}\right)
$$

Substituting into equation (2) about $\delta V$ from equation (4) we obtain the vacuum polarization correction in the $1 s$ state. To show the effect of pion form factor we compare $\delta E$ calculatedusing the pion as a point charge and that calculated in case of pion form factor from equation

Where

$$
\delta V(r)=\frac{4 \pi}{q^{2}} \int_{0}^{\infty} \frac{d^{3} q}{(2 \pi)^{3}} e^{-i \vec{q} \cdot \vec{r}} \Pi^{R}\left(-q^{2}\right) \rho_{N}(q) \rho_{\pi}(q)
$$

$$
\Pi^{R}\left(-q^{2}\right)=\frac{\alpha q^{2}}{\pi m^{2}} \int_{0}^{1} d v \frac{v^{2}\left(1-\frac{1}{3} v^{2}\right)}{1+\frac{q^{2}}{4 m^{2}}\left(1-v^{2}\right)} \approx-\frac{\alpha q^{2}}{15 \pi m^{2}}(\text { for small } \mathrm{q})
$$

In this work we take the nucleus distribution in the form

1- Fermi distribution

$$
\rho_{F}\left(r_{N}\right)=\frac{\rho_{o}}{1+e^{\left(\frac{r-C}{D}\right)}}
$$

Where $C$ is the root mean square radius of the nucleus and $D$ is the diffuseness.

2- Exponential distribution

$$
\rho_{E}\left(r_{N}\right)=\frac{\xi^{2}}{8 \pi} e^{-\xi r_{N}}
$$

Where $\xi=\sqrt{12 /\left\langle C^{2}\right\rangle}$ and the Fourier transform of the exponential distribution is 
Pion Form Factor Contribution in Vacuum Polarization corrections for 1s energy level in different

3- Gaussian distribution

$$
\rho_{N}(q)=\frac{1}{\left(1+R^{2} q^{2}\right)^{2}} \quad R=\frac{1}{\xi}
$$

And its Fourier transform is

$$
\rho_{G}\left(r_{N}\right)=\frac{\xi^{2}}{\pi^{3 / 2} a^{3}} e^{-(r / a)^{2}}
$$

$$
\rho_{\pi}(q)=e^{-a^{2} q^{2} / 4}
$$

\begin{tabular}{|c|c|c|c|c|c|c|c|c|}
\hline $\mathbf{Z}$ & \begin{tabular}{l}
\multicolumn{1}{c}{$\Delta E_{p D}$} \\
point pion and \\
point nucleus \\
distributions
\end{tabular} & \begin{tabular}{l}
\multicolumn{1}{c}{$\Delta E_{P C}$} \\
point pion and \\
Gaussian \\
nucleus \\
distributions \\
\end{tabular} & \begin{tabular}{l}
\multicolumn{1}{c}{$\Delta E_{P E}$} \\
point pion and \\
exponential \\
nucleus \\
distributions \\
\end{tabular} & \begin{tabular}{l}
\multicolumn{1}{c}{$\Delta E_{P F}$} \\
point pion and \\
Fermi nucleus \\
distributions
\end{tabular} & \begin{tabular}{lc}
\multicolumn{2}{c}{$\Delta E_{G D}$} \\
Gaussian pion \\
and & point \\
nucleus & \\
distributions \\
\end{tabular} & \begin{tabular}{l}
\multicolumn{1}{c}{$\Delta E_{G G}$} \\
Gaussian pion \\
and Gaussian \\
nucleus \\
distributions
\end{tabular} & \begin{tabular}{l}
\multicolumn{1}{c}{$\Delta E_{C E}$} \\
Gaussian pion \\
and exponential \\
nucleus \\
distributions
\end{tabular} & \begin{tabular}{l}
\multicolumn{1}{c}{$\Delta E_{G F}$} \\
Gaussian pion \\
and Fermi \\
nucleus \\
distributions
\end{tabular} \\
\hline 1 & $.184994 \times 10^{-7}$ & $-9.638224 \times 10^{-7}$ & $-5.938681 \times 10^{3}$ & $-6.97573 \times 10^{3}$ & $-1.365753 \times 10^{-7}$ & $-9.638224 \times 10^{-7}$ & $-3.390753 \times 10^{5}$ & $-2.595568 \times 10^{-6}$ \\
\hline 2 & $459026 \times 1$ & $-2.969949 x$ & $2.448216 \times 10^{5}$ & $.026425 x$ & $-1.636947 \times 10^{5}$ & $-2.969949 \times 10^{5}$ & $-5.303373 \times 10^{-5}$ & -2.291662 \\
\hline 3 & $621455 \times 10^{5}$ & -1.731938 & $933731 \times 10^{-5}$ & $148077 \times 10^{5}$ & $-4.708611 \times 10^{-5}$ & $-1.731938 \times 10^{-3}$ & $-1.636947 \times 10^{5}$ & $-1.163212 \times 10^{5}$ \\
\hline 4 & $64742 \times 10^{-4}$ & 684461 & $.869578 \times 10^{5}$ & $.702745 \times 10^{5}$ & $-8.415131 \times 10^{-5}$ & $-1.684461 \times 10^{-3}$ & $-1.497225 \times 10^{5}$ & $058994 \times 10^{-3}$ \\
\hline 5 & $99536 \times 10^{-4}$ & 7040 & $29495 \times 10^{-5}$ & $15414 \times 10^{-6}$ & $19274 \times 10^{-4}$ & $-1.870406 \times 10^{-5}$ & $-1.557707 \times 10^{5}$ & .120222 \\
\hline 6 & $3755 \times 10^{-4}$ & $0^{3}$ & $16399 \times 10^{-5}$ & $918824 \times 10^{5}$ & $45743 \times 10^{-4}$ & $-1.806508 \times 10^{5}$ & $-1.655799 \times 10^{5}$ & 60564 \\
\hline 7 & $23948 \times 10^{-4}$ & $1.680234 \times 10^{5}$ & $.158824 \times 10^{5}$ & $.498896 \times 10^{5}$ & $2.088948 \times 10^{-4}$ & $-1.680234 \times 10^{5}$ & $-1.699478 \times 10^{-5}$ & $815202 \times 10^{5}$ \\
\hline 8 & $.75669 \times 10^{-4}$ & $1.896082 \times 10^{-5}$ & $.426381 \times 10^{5}$ & $5.684951 \times 10^{5}$ & $-2.484432 \times 10^{-4}$ & $-1.896082 \times 10^{5}$ & $-1.733258 \times 10^{5}$ & $083291 \times 10^{-6}$ \\
\hline 9 & $.339532 \times 10^{-3}$ & $-1.150806 \times 10^{5}$ & $7014 \times 10^{-5}$ & $-2.201174 \times 10^{-6}$ & $0166 \times 10^{-4}$ & $-1.150806 \times 10^{5}$ & $-1.736319 \times 10^{-5}$ & $950966 \times 10^{6}$ \\
\hline 10 & $7928 \times 10^{-3}$ & & $-1.19772610^{\circ}$ & $-1.269972 \times 10^{5}$ & $-3.446901 \times 10^{-4}$ & $-1.047206 \times 10^{3}$ & $-1.752557 \times 10^{3}$ & $-6.38844 \times 10^{-6}$ \\
\hline 11 & $-2.322134 \times 10^{3}$ & $-1.135836 \times 10^{5}$ & $-1.357885 \times 10^{5}$ & $-9.717939 \times 10^{-7}$ & $-3.841923 \times 10^{-4}$ & $-1.135836 \times 10^{5}$ & $-1.804106 \times 10^{-5}$ & $-6.884018 \times 10^{5}$ \\
\hline 12 & $-2.954968 \times 10^{3}$ & $-1.12591 \times 1$ & $-1.435044 \times 10^{5}$ & $-5.852169 \times 10^{-7}$ & $-4.248391 \times 10^{-4}$ & $-1.12591 \times 10^{-5}$ & $-1.903543 \times 10^{5}$ & $-6.846243 \times 10^{6}$ \\
\hline 15 & $-5.516989 \times 10^{-3}$ & & $-1.61992 \times 10^{-5}$ & $-9.33944 \times 10^{-3}$ & $-5.447423 \times 10^{-4}$ & $-1.096805 \times 10^{-5}$ & $-1.98158 \times 10^{-5}$ & $-6.747359 \times 10^{-6}$ \\
\hline 17 & $6989 \times 10^{3}$ & $0^{5}$ & $1992 \times 10^{-6}$ & $-9.33944 \times 1$ & $243065 \times 10^{-4}$ & $832603 \times 10^{5}$ & $-2.221527 \times 10^{-3}$ & $-6.149533 \times 10^{-6}$ \\
\hline 18 & $4413 \times 10^{-3}$ & & $87378 \times 10^{-5}$ & $-8.067143 \times 10^{-9}$ & $629033 \times 10^{-4}$ & $015883 \times 10^{-5}$ & $-2.364526 \times 10^{-3}$ & $-6.354092 \times 10^{5}$ \\
\hline 19 & & & $34954 \times 10^{3}$ & 29672 & $16468 \times 10^{-4}$ & $10^{5}$ & $53365 \times 10^{-3}$ & $387836 \times 10^{5}$ \\
\hline 23 & & & $484 x$ & & $7152 x$ & & $35763 \times 10^{5}$ & $54237 \times 10^{-6}$ \\
\hline 26 & & & $0^{5}$ & -22 & $3626 x$ & $r^{3}$ & $1513 x$ & $10^{-5}$ \\
\hline 27 & 0215 & $\overline{0^{5}}$ & & -12 & $8779 x$ & $10^{6}$ & $76286 \times 10^{5}$ & $\times 10^{5}$ \\
\hline 28 & 3069 & $1662 \times 10^{5}$ & $598410^{-2}$ & $0^{-12}$ & $6714 x$ & $52 \times 10^{-5}$ & $38577 \times 10^{-5}$ & $94563 \times 10^{6}$ \\
\hline 30 & & & $3039 \times 10^{-5}$ & & $0^{3}$ & $10^{5}$ & $11769 \times 10^{5}$ & $\mathrm{x} 10^{-6}$ \\
\hline 32 & 367 & $16944 \times 10^{-5}$ & $68187 \times 10^{-5}$ & $7161 \times 10^{-15}$ & $199239 \times 10^{3}$ & $.216944 \times 10^{5}$ & $3.332592 \times 10^{-3}$ & $-6.550851 \times 10^{-5}$ \\
\hline 36 & 422 & $58116 \times 10^{-6}$ & $101864 \times 10^{5}$ & $83076 \times 10^{-16}$ & $350792 \times 10^{3}$ & $-9.558116 \times 10^{6}$ & $445233 \times 10^{5}$ & $-6.84299 \times 10^{-6}$ \\
\hline 37 & & & $35674 \times 10^{3}$ & $-7.02101 \times 10^{-17}$ & $388643 \times 10^{3}$ & $\times 10^{5}$ & $653905 \times 10^{5}$ & $18253 \times 10^{6}$ \\
\hline 41 & 09574 & $90695 \times 10^{5}$ & $89118 \times 10^{5}$ & $-9.08101 \times 10^{-25}$ & $-1.540058 \times 10^{-3}$ & $-9.590695 \times 10^{5}$ & $-3.698067 \times 10^{-5}$ & $-6.926199 \times 10^{5}$ \\
\hline 42 & -0.117614 & $06418 \times 10^{-5}$ & $-2.24399 \times 10^{-5}$ & $-4.06086 \times 10^{-2}$ & $-1.577834 \times 10^{3}$ & $-9.806418 \times 10^{6}$ & $-3.833687 \times 10^{5}$ & $-7.083369 \times 10^{-6}$ \\
\hline 45 & -0.153439 & $-9.158592 \times 10^{6}$ & $-2.163247 \times 10^{5}$ & $-6.78788 \times 10^{-21}$ & $-1.69141 \times 10^{-3}$ & $-9.158592 \times 10^{-6}$ & $-3.860234 \times 10^{5}$ & $-6.671895 \times 10^{5}$ \\
\hline 48 & 93001 & & $5928 \times 10^{5}$ & $1523 \times 10^{-22}$ & $04761 \times 10^{3}$ & $88943 \times 10^{-6}$ & $-3.901468 \times 10^{5}$ & $-6.7878 \times 10^{5}$ \\
\hline 49 & 09495 & $-9.217895 \times 10^{5}$ & $-2.223835 \times 10^{3}$ & $-9.90286 \times 10^{-23}$ & $-1.842561 \times 10^{-3}$ & $-9.217895 \times 10^{5}$ & $-3.907672 \times 10^{5}$ & $-6.746978 \times 10^{6}$ \\
\hline 53 & -0.291666 & $-9.043298 \times 10^{5}$ & $-2.233198 \times 10^{-3}$ & $-1.06639 \times 10^{-24}$ & $-1.993737 \times 10^{3}$ & $-9.043298 \times 10^{6}$ & $-3.898346 \times 10^{-3}$ & $-6.65574 \times 10^{-6}$ \\
\hline 54 & -0.31675 & $-9.081185 \times 10^{5}$ & $-2.25138 \times 10^{-5}$ & $-3.94558 \times 10^{-25}$ & $-2.031524 \times 10^{3}$ & $-9.081185 \times 10^{5}$ & $-3.80294 \times 10^{-5}$ & $-6.6891 \times 10^{-6}$ \\
\hline 55 & & $-9.145242 \times 10^{6}$ & $-2.274821 \times 10^{-3}$ & $-1.53375 \times 10^{-35}$ & $-2.069313 \times 10^{-3}$ & $-9.145242 \times 10^{6}$ & $-3.763212 \times 10^{-5}$ & $-6.74065 \times 10^{-6}$ \\
\hline 57 & & $-9.10452 \times 10^{-5}$ & $-2.286509 \times 10^{-3}$ & $-1.79003 \times 10^{-36}$ & $-2.145834 \times 10^{-3}$ & $-9.10452 \times 10^{-6}$ & $-3.716263 \times 10^{-3}$ & $-6.725181 \times 10^{-6}$ \\
\hline 59 & & $-9.282709 \times 10^{-6}$ & $-2.343204 \times 10^{3}$ & $-3.06826 \times 10^{-27}$ & $-2.219964 \times 10^{-3}$ & $-9.282709 \times 10^{6}$ & $-3.597389 \times 10^{-3}$ & $-6.862737 \times 10^{5}$ \\
\hline 60 & -0.558409 & $-9.249544 \times 10^{5}$ & $-2.346062 \times 10^{-3}$ & $-1.03965 \times 10^{-27}$ & $-2.258472 \times 10^{3}$ & $-9.249544 \times 10^{-6}$ & $-3.44238 \times 10^{-3}$ & $-6.845627 \times 10^{-6}$ \\
\hline 66 & -1.609142 & $-8.618535 \times 10^{6}$ & $-2.265432 \times 10^{-3}$ & $-7.42035 \times 10^{-31}$ & $-2.484606 \times 10^{-3}$ & $-8.618535 \times 10^{6}$ & $-2.454152 \times 10^{-5}$ & $-6.43075 \times 10^{-6}$ \\
\hline 68 & & $-8.631953 \times 10^{-6}$ & $-2.287854 \times 10^{-3}$ & $-8.22535 \times 10^{-32}$ & $-2.560285 \times 10^{-3}$ & $-8.631953 \times 10^{5}$ & $-1.829735 \times 10^{-6}$ & $-6.449571 \times 10^{5}$ \\
\hline
\end{tabular}

Table 1. Vacuum Polarization corrections for the energy level 1s (in MeV) of the pionic-hydrogen atoms calculated by applying Lee [5] method for the spin zero pion particle.

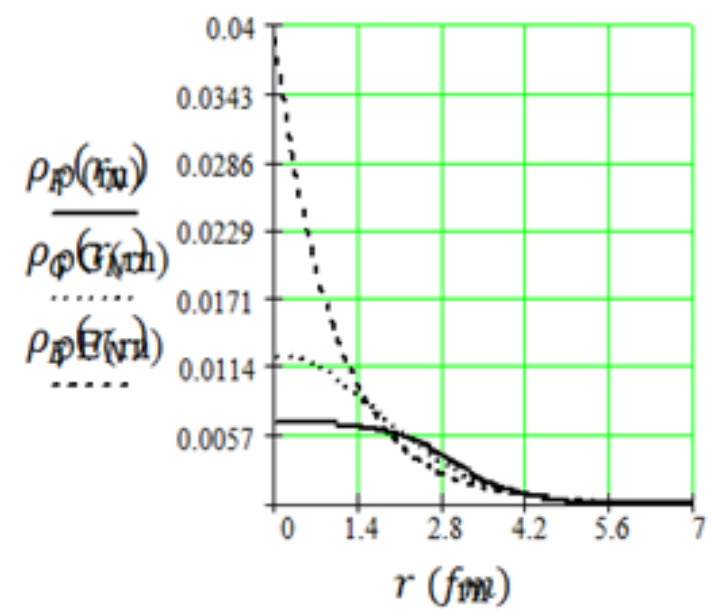

Fig.2 Three spherical symmetric charge densities ofthe nucleus, for $Z=11$ 


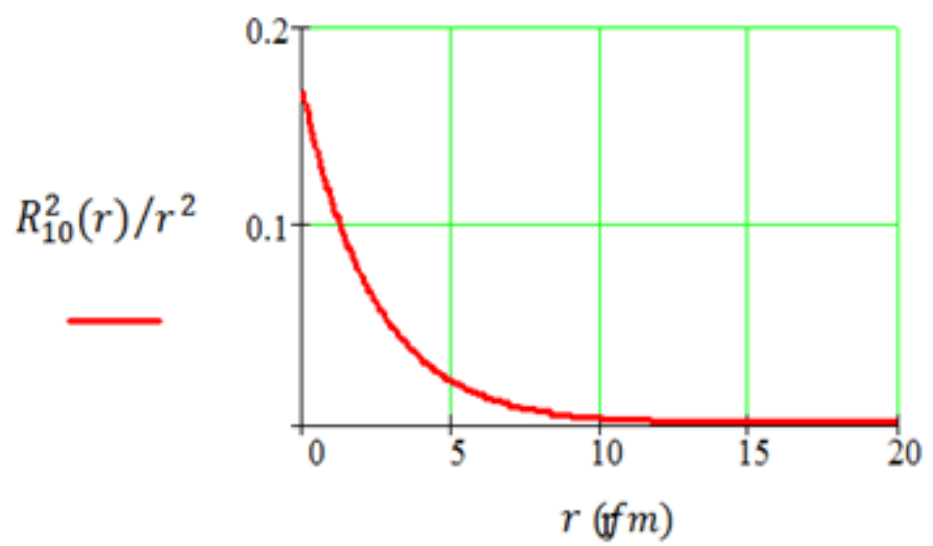

Fig.3 Represents the 1s square of the radial unperturbed wave function $R_{10}^{2}(r) / r^{2}$ obtained from equation (1).

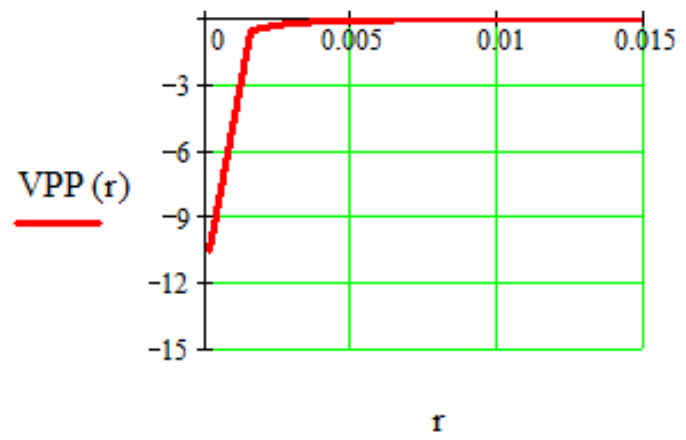

(a)Represents the potential in case of point nucleus with point pion.

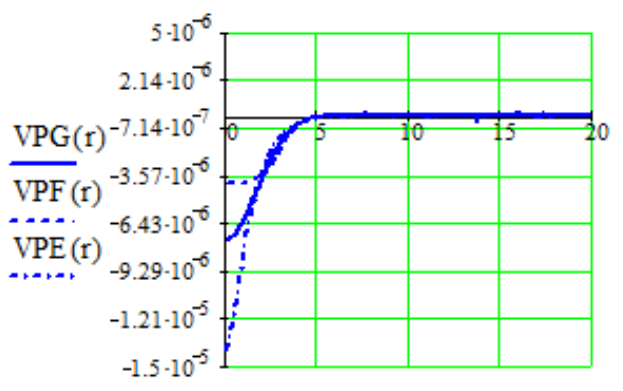

(b) Represents the pion as a point and the nucleus takes three forms of form factors. The dotted line is Gaussian, the solid line is Fermi and the dashed is exponential.

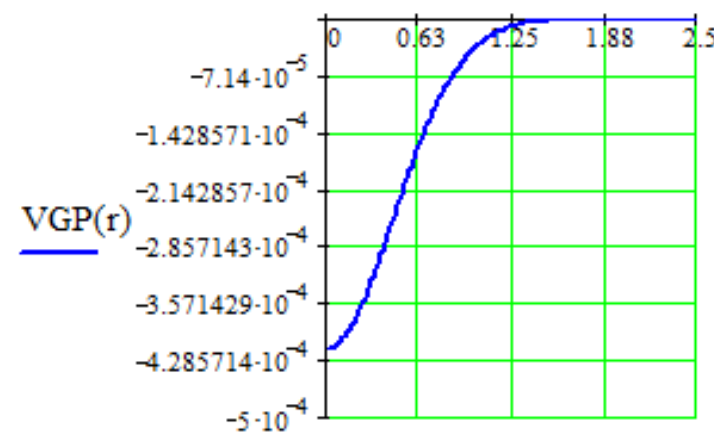

$\mathrm{r}$

(c) Represents the potential in case of point nucleus with Gaussian form factor of pion. 


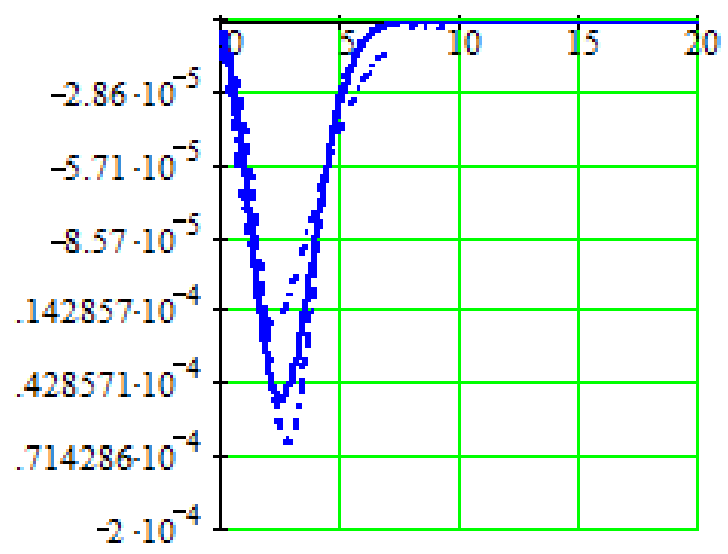

r

(d) Represents the potential in case the pion as Gaussian and the nucleus takes three forms of form factors. The solid line is Gaussian, the dashed is Fermi and the dash-dot is exponential.

Fig.4 The figure represents different forms of the potential for $\mathrm{Z}=11$ as an example.

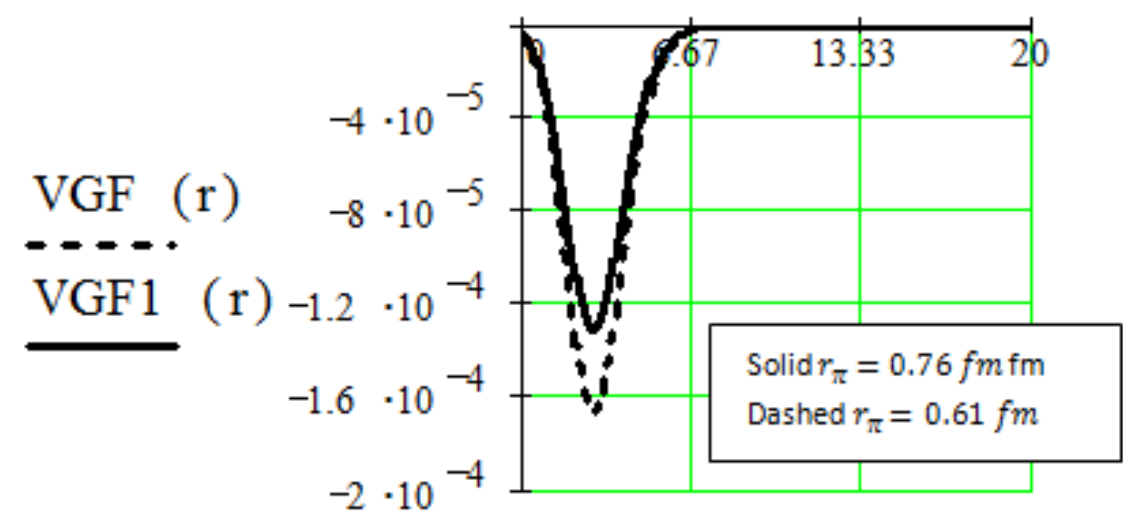

Fig.5 The effect of the pion radius on the potential of fermi distributed nucleus and Gaussian pion form factor.

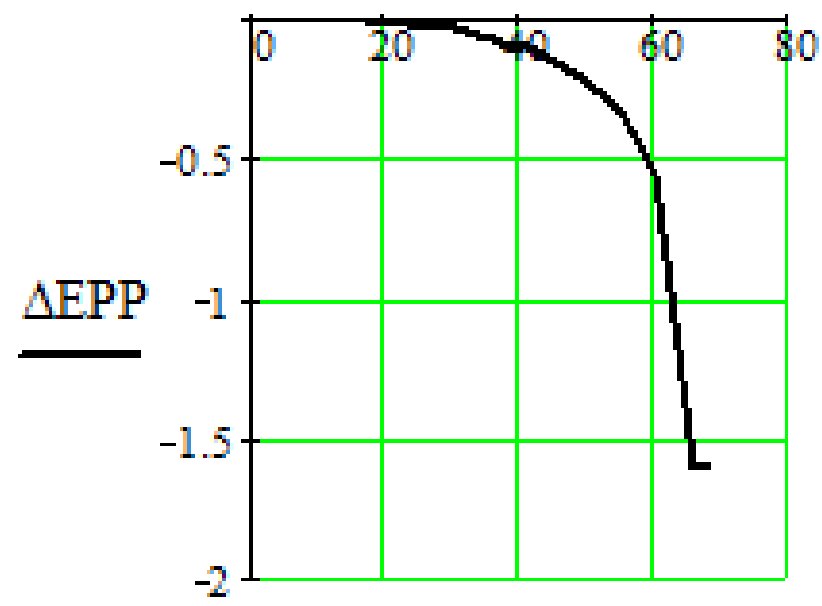

Z

(a) Represents the pion as a point and the nucleus as a point. 


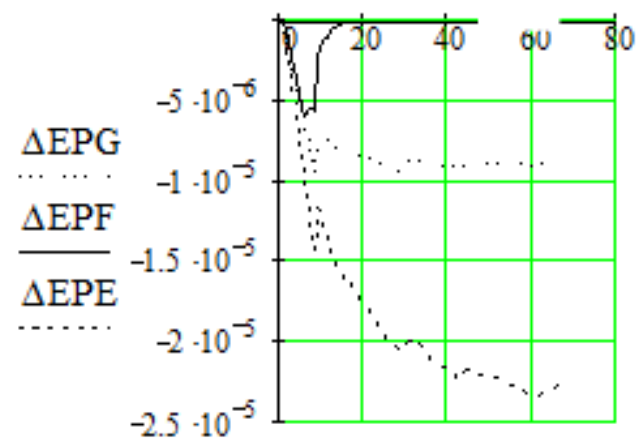

Z

(b) Represents the pion as a point and the nucleus takes three forms of form factors. The dotted line is Gaussian, the solid line is Fermi and the dashed is exponential.

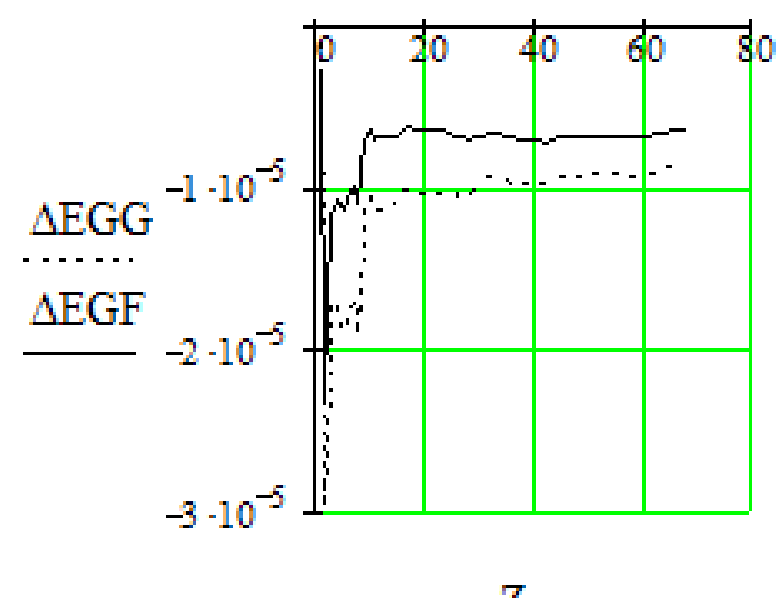

(c) Represents the pion as Gaussian and the nucleus takes two forms of form factors. The dotted line is Gaussian, while the solid line is Fermi.

Fig.6 Represents the vacuum polarization corrections for the 1 s energy level for different $\mathrm{Z}$ pionic-hydrogen atoms.

\section{Results And Discussion}

In these calculations we use the relativistic units $\hbar=c=1$, the pion mass $m_{\pi}=139.577 \mathrm{MeV}$, the electron mass $m_{e}=0.5109989 \mathrm{MeV}$ and the fine structure constant $\alpha=1 / 137.0359998$, and the radius of pion is $0.61 \pm 0.15 \mathrm{fm}$ [9]. Figure 2 shows the three different distributions mentioned above. In the short range the exponential density is higher than the Gaussian density and the Gaussian is higher than the Fermi distribution. For higher distances they are approximately the same. Figure 3 represents the $1 s$ square of the unperturbed radial wave function of pion obtained from equation (1) against $r$, for $Z=11$ and radius $2.986 \mathrm{fm}$ [10] and reduced mass $19.738 \mathrm{MeV}$. From the figure it is clear that most contribution of the wave function is found at the origin, so that the potential which has large values at the origin will give a large correction to the energy.From figures 4-a,4-b, and 4-c it is clear that these potentials have large overlap with the wave function because the maximum exists at the origin, like the wave function. The point nucleus potential with Gaussian form factor, fig. 4-c, has the shortest range $(1.2 \mathrm{fm})$, while the pion point potential with Gaussian, Fermi, and exponential form factor of the nucleus has a range of about $5 \mathrm{fm}$. Figure 4-d has three maxima for the three potentials. The potential with the Fermi nucleus and Gaussian pion form factor has the highest peak. The potential with exponential nucleus distribution and pion Gaussian distribution has the lowest peak. These peaks are not at the origin hence the values of the corrections corresponding to these three potentials are smaller than the other potentials because the overlap of these potentials with the wave function is small. The position of these peaks is as follows VGF at $r=2.749 \mathrm{fm}$, VGG at $r=2.473 \mathrm{fm}$, and VGE at $r=2.113 \mathrm{fm}$. To show the effect of the pion radius on the potential, figure 5 is drawn. From the figure it is clear that the peak is lowered when the radius of pion increases. For the energy the increase in radius of pion decreases the value of the vacuum polarization correction, e.g. for $Z=11, \Delta E=-6.476599 \times 10^{-6} \mathrm{MeV}$ for pion radius $0.76 \mathrm{fm}$ and 
$\Delta E=-6.884018 \times 10^{-6} \mathrm{MeV}$ for pion radius $0.61 \mathrm{fm}$. From figure 6 and table 1 , we can conclude that without form factor of nucleus and pion the correction increases very slowly for low $Z$ atoms and increases fastly for higher $Z$. The finite size of the nucleus increases the correction with $Z$ in case of exponential distribution, while in case of Gaussian distribution the increase is lower. For Fermi distribution there is a fast increase at low values of $Z$ and faslty decreases with higher values of $Z$. The effect of form factor of pion on the correction is very clear for low $Z$ nuclei and then becomes nearly constant for higher values $Z$.

\section{Acknowledgment}

The first author would like to thank Professor A. Faessler and professor W. Griener for their help and encouraging.

\section{References}

[1]. M El shabshiry, S.M.E. Ismaeel, and M.M. Abdel-Mageed, IOSR Jornal of applied Physics, e-ISSN: 2278-4861. Volume 7, Issue 5 Ver. I. 2015, pp 60-66.

[2]. M El shabshiry, S.M.E. Ismaeel, and M.M. Abdel-Mageed, IOSR Jornal of applied Physics, e-ISSN: 2278-4861. Volume 8, Issue 1 Ver. III. 2016, pp 47-53.

[3]. E. Borie, Phys. Rev. A71 032508 (2005)

[4]. J.D. Carroll, A.W. Thomas, J.Rafelski and A.G. Miller, Phys. Rev. A84 012506 (2011).

[5]. R.N. Lee, A. I. Milstein and S. G. karshenboim, Phys. Rev. A73 012505 (2006)

[6]. S.G. Karshenboim, E. Yu. Korzinin and V.G. Ivanov, Canadian Journal of Physics 84 (2) 107-113 (2006).

[7]. V.S. Popov, Sov. Jour. Nucl. Phys. 12, 235 (1971).

[8]. W. Fleischer and G. Soff, Z. Naturforschung 101, 703 (1984).

[9]. G. T. Adylovet. al. Phys. Lett. B, Vol. 51, Issue 4, page 402-406 (1974).

[10]. B A Brown, C R Bronk and P E Hodgson, J. Phys. G. Nucl. Phys. 10 (1984) 1683-1701. Printed in Great Britain. 